\author{
Vincenzo Cantaluppi \\ Barbara Assenzio \\ Daniela Pasero \\ Giuseppe Mauriello Romanazzi \\ Alfonso Pacitti \\ Giacomo Lanfranco \\ Valeria Puntorieri \\ Erica L. Martin \\ Luciana Mascia \\ Gianpaola Monti \\ Giampaolo Casella \\ Giuseppe Paolo Segoloni \\ Giovanni Camussi \\ V. Marco Ranieri
}

\title{
Polymyxin-B hemoperfusion inactivates circulating proapoptotic factors
}

Received: 21 November 2007

Accepted: 2 April 2008

Published online: 8 May 2008

(c) The Author(s) 2008

Supported by Italian Ministry of University and Research (COFIN 4657/06-08),

Regione Piemonte (integrated project A47, Ministry of Health (Ricerca Finalizzata 02), and Fondazione S. Paolo (Progetto S.

Paolo).

Barbara Assenzio, Vincenzo Cantaluppi, and Daniela Pasero equally contributed to the manuscript and should all be considered as first author.

Electronic supplementary material The online version of this article (doi: 10.1007/s00134-008-1124-6) contains supplementary material, which is available to authorized users.

\footnotetext{
V. Cantaluppi - G. M. Romanazzi ·

G. Camussi

Dipartimento di Medicina Interna, Centro Ricerca Medicina Sperimentale (CeRMS), Torino, Italy

V. Cantaluppi - A. Pacitti - G. Lanfranco . G. P. Segoloni - G. Camussi

Ospedale S. Giovanni Battista-Molinette, SCDU Nefrologia, Dialisi e Trapianti Università di Torino, Torino, Italy
}

B. Assenzio - D. Pasero - V. Puntorieri · E. L. Martin · L. Mascia •

V. M. Ranieri (®)

Dipartimento di Anestesiologia e

Rianimazione, Ospedale S. Giovanni

Battista-Molinette, Università di Torino,

Corso Dogliotti 14, 10126 Torino, Italy

e-mail: marco.ranieri@unito.it

Tel.: +39-11-6334001

Fax: +39-11-6960448

\section{G. Monti - G. Casella}

Ospedale Niguarda, Servizio di Anestesia e Rianimazione, Milano, Italy

Abstract Objective: To test the hypothesis that extracorporeal therapy with polymyxin B (PMX-B) may prevent Gram-negative sepsisinduced acute renal failure (ARF) by reducing the activity of proapoptotic circulating factors. Setting: Medical-Surgical Intensive Care Units. Patients and interventions: Sixteen patients with Gram-negative sepsis were randomized to receive standard care (Surviving Sepsis Campaign guidelines) or standard care plus extracorporeal therapy with PMX-B. Measurements and results: Cell viability, apoptosis, polarity, morphogenesis, and epithelial integrity were evaluated in cultured tubular cells and glomerular podocytes incubated with plasma from patients of both groups. Renal function was evaluated as SOFA and RIFLE scores, proteinuria, and tubular enzymes. A significant decrease of plasma-induced proapoptotic activity was observed after PMX-B treatment on cultured renal cells. SOFA and RIFLE scores, proteinuria, and urine tubular enzymes were all significantly reduced after PMX-B treatment. Loss of plasma-induced polarity and permeability of cell cultures was abrogated with the plasma of patients treated with PMX-B. These results were associated to a preserved expression of molecules crucial for tubular and glomerular functional integrity. Conclusions: Extracorporeal therapy with PMX-B reduces the proapoptotic activity of the plasma of septic patients on cultured renal cells. These data confirm the role of apoptosis in the development of sepsisrelated ARF.

Keywords Sepsis - Acute renal failure - Lipopolysaccharide · Apoptosis 


\section{Introduction}

Acute renal failure (ARF) occurs in $20 \%$ of patients with severe sepsis and in the $50 \%$ of patients with septic shock $[1,2]$. The combination of ARF and sepsis is associated with a $70 \%$ mortality rate as compared with a $45 \%$ mortality rate in patients with ARF alone [1,2].

Experimental [3-5] and clinical [6] studies suggested that circulating lipopolysaccharide (LPS), a cell wall component of Gram-negative bacteria, is the key element of the sepsis-induced ARF [7]. Direct hemoperfusion through a column of immobilized polymyxin B (PMX-B) to reduce plasma levels of LPS [8-10] has been therefore proposed to improve hemodynamics and oxygenation [11] and prevent ARF [12].

Apoptosis, an energy-dependent process whereby cells carry out programmed death [13], contributes to the pathogenesis of ARF [14]. Recently, Jo et al. [4] and Bordoni et al. [15] suggested that Fas-mediated and caspase-mediated apoptosis of tubular cells might be one of the possible mechanisms involved in the endotoxemiainduced renal dysfunction. Consistent with these findings, several studies have shown that circulating LPS may cause an inappropriate activation of proapoptotic pathways in immune, epithelial, and endothelial cells $[6,16$, 17]. Moreover, LPS can directly act on kidney-resident cells such as podocytes and tubular epithelium, stimulating the synthesis of inflammatory mediators [18-20].

The present study was designed to test the hypothesis that extracorporeal therapy with PMX-B may prevent Gram-negative sepsis-induced ARF by reducing the activity of proapoptotic circulating factors on tubular cells and glomerular podocytes. The primary outcome variable was viability of renal cell cultures exposed to the plasma of septic patients. Secondary outcome variables were different markers of renal function. Parts of these data were published as an abstract [21].

\section{Methods}

Patients admitted to the intensive care units of the Molinette (University of Turin) and Niguarda (Milan) hospitals were recruited between January 2006 and April 2007. Ethics committees approved the protocol and written informed consent was obtained from all patients. A detailed version of the methods is available in the Electronic Supplementary Material (ESM).

\section{Patients and treatments}

Patients were eligible to participate in the study if they had a positive culture for Gram-negative bacteria and randomization could be performed within $24 \mathrm{~h}$ of matching study criteria. Inclusion criteria were as follows: (1) three of the systemic inflammatory response system (SIRS) and (2) presence of one organ dysfunction [22, 23]. Exclusion criteria were as follows: (1) presence of two or more failing organs; life expectancy $\leq 30$ days; (2) presence of a "do not resuscitate" order; (3) HIV infection; (4) uncontrolled hemorrhage within $24 \mathrm{~h}$ before study entry; (5) organ transplantation during the year before study entry; (6) history of sensitivity to polymyxinB or anticoagulant and/or extracorporeal circulation; (7) severe thrombocytopenia $\left(<30,000 \mathrm{cells} / \mathrm{mm}^{3}\right)$ and/or granulocytopenia $\left(<500\right.$ cells $\left./ \mathrm{mm}^{3}\right)$; (8) an acute physiology and chronic health evaluation (APACHE) II score $>30$ [23].

Patients were randomized to receive conventional treatments according to the Surviving Sepsis Campaign guidelines [24] (CONV group) or to undergo, in addition to standard care, a hemoperfusion session with Polymyxin-B Fiber cartridge at the moment of inclusion and after $24 \mathrm{~h}$. Each hemoperfusion session lasted $2 \mathrm{~h}$ (PMX group). Sequential organ failure assessment (SOFA) [25] and Acute-Dialysis-Quality Initiative (ADQI)-RIFLE [scoring system that defines three grades of increasing severity of ARF (risk, injury, and failure, respectively, R, $\mathrm{I}$, and F) and two outcome variables (loss and end-stage kidney disease, respectively, L and E)] [26] scores were evaluated at T0 and T72. Amount and kind of catecholamine support were also recorded at $\mathrm{T} 0$ and $\mathrm{T} 72$.

\section{Plasma and urine determinations}

Blood and urine samples were collected at the time of inclusion (T0) and $24 \mathrm{~h}$ (T24) and $72 \mathrm{~h}$ (T72) later. In the PMX group, samples were also collected at the end of each hemoperfusion (T2 and T26). Clinical variables were followed for 28 days. Blood samples were stored at $-80^{\circ} \mathrm{C}$. Plasma levels of LPS were evaluated by Limulus Amebocyte Lysate (LAL) kinetic test (Cambrex, Walkersville, MD). Plasma concentrations of tumor necrosis factor- $\alpha$ (TNF- $(\alpha)$ were determined by an enzyme-linked immunoabsorbent assay (ELISA; R\&D Systems, Minneapolis, MN). Urine samples were collected for the evaluation of proteins and the tubular enzymes NAG $(N$ acetyl-glucosaminidase), GGT (gamma-glutamyl transpeptidase), and AAP (Ala-Leu-Gly aminopeptidase). All values were expressed as ratio to urine creatinine [17] to avoid the tubular concentration factor [27].

\section{Kidney cell cytotoxicity and apoptosis}

Human proximal tubular epithelial cells and glomerular podocytes were isolated from the renal cortex and cultured as previously described [28]. Cells were incubated with plasmas obtained from the CONV group and the 
PMX group. Plasma from healthy volunteers or vehicle alone was used as controls. The cytotoxic effect of different plasmas was evaluated by a colorimetric assay based on the ability of XTT sodium salts to bind to mitochondrial dehydrogenases of viable cells [29]. Podocytes and tubular cell cultures were subjected to terminal deoxynucleotidyltransferase (TdT)-mediated dUTP nick end labeling (TUNEL) assay [29] to identify chromatin fragmentation in apoptotic cells. In a pilot study, that included five patients that matched study inclusion and exclusion criteria, we found that the collected plasma induced apoptosis in 45-50 cells per field of cultured tubular and podocytes. This trial was hence designed to enroll 16 patients to demonstrate at least a $30 \%$ reduction in proapoptotic activity, with a 5\% risk of type I error and a power of 90\%. Fas (CD95) was detected by immunofluorescence and by FACS analysis as previously described [4]. Bax and Bcl-2 family proteins were investigated by Western blot [29]. The activity of caspase-3, caspase-8, and caspase- 9 was assessed by ELISA.

In selected experiments, tubular cells were seeded on six-well plates and TNF-receptor 1 (TNF-R1) short interfering RNA (siRNA) or relative control siRNA $(80 \mathrm{pM})$ was introduced according to manufacturer's instructions (Santa Cruz Biotech., Santa Cruz, CA). After $48 \mathrm{~h}$, TNF-R1 knockdown was verified by RT-PCR, immunofluorescence, and Western blot analysis. Subsequently, engineered tubular cells were incubated with different plasma and subjected to TUNEL assay.

\section{Kidney cell functions}

Transepithelial electrical resistance (TER) was used to estimate alteration of selective cell polarity [30]. Permeability was evaluated by diffusion of Trypan blue-albumin complexes on podocyte monolayer. Adhesion to extracellular matrixes and morphogenesis assay was studied as previously described [30]. Expression of intercellular adhesion molecules-1 (ICAM-1) and CD40 on tubular epithelial cells was evaluated by cytofluorimetric analysis. Detection of ZO1, megalin, nephrin, and B7.1 was performed by immunofluorescence [31, 32].

\section{Statistical analysis}

The data were expressed as mean $\pm \mathrm{SD}$ or median and range when appropriate. Comparisons were performed using the unpaired $t$ test, the Mann-Whitney, the chisquare and the Fisher exact test when appropriate. Comparison among different time points within each group was evaluated using a two-way analysis of variance for repeated measurements (ANOVA) with Newman-Keuls correction; if significant $(P<0.05)$, values obtained at different levels of exposure to plasma were compared with T0 using the paired $t$ test or Wilcoxon's signed-rank test when appropriate (Statview 5.0, CA).

\section{Results}

Twenty patients with Gram-negative infection and sepsis were assessed for eligibility. One was excluded for HIV infection, three for presence of two or more failing organs. Sixteen consecutive patients were enrolled, eight patients in the PMX-B group and eight in the CONV group. Some of the results are available on the ESM.

\section{Effects on clinical and biochemical parameters}

On admission, all patients were treated according to the Surviving Sepsis Campaign guidelines and required catecholamine support. Patients were equally distributed for age, gender, APACHE II, sites of infection, antibiotic therapy and organ dysfunctions (Table 1). After $72 \mathrm{~h}$, SOFA decreased $(P=0.02)$ and RIFLE improved $(P=0.04)$ in the PMX group, while it did not change in the CONV group; five out of eight patients were weaned from catecholamine support in the PMX group $(P=0.02)$, whereas in the CONV group all patients but one were still on catecholamines (Table 1). The need for renal replacement therapy occurred in one patient in the PMX group (17\%) and in three patients $(37 \%)$ in the CONV group $(P=0.02)$. In the PMX group but not in CONV group, a significant reduction in plasmatic levels of LPS and TNF-alpha was observed at T72.

\section{Effect on cellular cytotoxicity and apoptosis}

Compared to incubation with vehicle alone or healthy plasma, septic plasma (concentration 5\% diluted in normal culture medium) derived from both PMX and CONV groups reduced the viability of glomerular podocytes (not shown) and tubular cells, as detected by the XTT-based assay (Figure 1, A and B in ESM). In the PMX group, a significant reduction of tubular injury was observed after challenging with plasma collected at the end of both PMX-B hemoperfusion sessions (PMX T2 and PMX T26) and after $72 \mathrm{~h}$ of the enrollment in the study (PMX T72). By contrast, in the CONV group, plasma-induced tubular injury remained constant over time (Figure 1, A and B in ESM).

Plasma from patients of CONV T0 and PMX T0 groups induced significant tubular cell apoptosis, as detected by TUNEL assay (Fig. 1a). While plasma-induced apoptosis remained significantly higher in the CONV T72 group, it was significantly reduced in the PMX T72 group compared to PMX T0. Furthermore, addition of $5 \mu \mathrm{g} / \mathrm{ml}$ PMX-B, a 
Table 1 Patients' demographic, clinical, and biochemical data

\begin{tabular}{|c|c|c|}
\hline & PMX & CONV \\
\hline Age (years) & $61 \pm 10$ & $59 \pm 13$ \\
\hline Male $N(\%)$ & $6(75)$ & $6(75)$ \\
\hline APACHE II & $21.2 \pm 3.6$ & $20.4 \pm 4.4$ \\
\hline \multicolumn{3}{|c|}{ Site of infection $N(\%)$} \\
\hline Abdominal & $7(87)$ & $5(65)$ \\
\hline Urinary tract & $1(13)$ & $3(35)$ \\
\hline \multicolumn{3}{|l|}{ Antibiotics $N(\%)$} \\
\hline Glycopeptides & $4(50)$ & $5(62)$ \\
\hline Aminoglycosides & $3(37)$ & $4(50)$ \\
\hline \multicolumn{3}{|l|}{ SOFA score } \\
\hline T0 & $11(13-7)$ & $9(20-7)$ \\
\hline $\mathrm{T} 72$ & $5.5(11-2)^{\mathrm{a}}$ & $10.5(23-6)^{\mathrm{d}}$ \\
\hline \multicolumn{3}{|l|}{ RIFLE $N(\%)$} \\
\hline \multicolumn{3}{|l|}{ Risk } \\
\hline T0 & $5(62.5)$ & $5(62.5)$ \\
\hline T72 & $2(25)$ & $2(25)$ \\
\hline \multicolumn{3}{|l|}{ Injury } \\
\hline T0 & $2(25)$ & $1(12.5)$ \\
\hline $\mathrm{T} 72$ & 0 & $1(12.5)$ \\
\hline \multicolumn{3}{|l|}{ Failure } \\
\hline T0 & 0 & 0 \\
\hline $\mathrm{T} 72$ & 0 & $1(12.5)$ \\
\hline \multicolumn{3}{|c|}{ Risk + injury + failure } \\
\hline T0 & $7(87)$ & $6(75)$ \\
\hline $\mathrm{T} 72$ & $2(25)^{b}$ & $4(50)$ \\
\hline \multicolumn{3}{|c|}{$\begin{array}{l}\text { Patients on catecholamine } \mathrm{N} \\
(\%)\end{array}$} \\
\hline T0 & $8(100)$ & $8(100)$ \\
\hline $\mathrm{T} 72$ & $3(37)^{c}$ & $7(87)$ \\
\hline \multicolumn{3}{|c|}{ Noradrenaline $(\mu \mathrm{g} / \mathrm{kg} / \mathrm{min})$} \\
\hline T0 & $0.5(1.0-0.1)$ & $0.45(0.8-0.08)$ \\
\hline $\mathrm{T} 72$ & $0(0.4-0)^{\mathrm{a}}$ & $0.12(0.8-0)$ \\
\hline \multicolumn{3}{|l|}{ LPS (pg/ml) } \\
\hline T0 & $187.83 \pm 21.45$ & $162.57 \pm 37.87$ \\
\hline $\mathrm{T} 72$ & $87.74 \pm 19.45^{\mathrm{a}}$ & $184.63 \pm 30.56$ \\
\hline \multicolumn{3}{|l|}{ TNF-alpha (pg/ml) } \\
\hline T0 & $84.53 \pm 19.85$ & $76.53 \pm 16.32$ \\
\hline $\mathrm{T} 72$ & $21.34 \pm 7.98^{\mathrm{a}}$ & $95.34 \pm 19.64$ \\
\hline
\end{tabular}

Data are expressed as mean \pm SD and median (range)

$P M X$ polymyxin-B, CONV conventional, APACHE II acute physiology and chronic health evaluation, SOFA sequential organ failure assessment, RIFLE risk, injury, failure, loss of function, end stage

${ }^{a}$ Wilcoxon Signed Rank test PMX: T0 versus T72, $P=0.02$

${ }^{b}$ Fisher Exact test PMX: T0 versus T72, $P=0.04$

c Fisher Exact test: PMX versus CONV at T72, $P=0.02$

${ }^{\mathrm{d}}$ Mann-Whitney test: PMX T72 versus CONV T72, $P=0.02$

dose found to significantly inhibit LPS biological activity without inducing tubular cell death, to the culture significantly reduced the proapoptotic activity of PMX T0, CONV T0, and CONV T72 plasma but not of PMX T72 plasma. However, plasma from the PMX-B hemoperfusion with or without the addition of Polymyxin-B did not completely abrogate the proapoptotic effect of plasma (Figure $1, \mathrm{C}$ in ESM). In all apoptosis experiments, LPS (30 ng/ml) was used as a positive control.

TNF-R1 knockdown by siRNA induced a significant decrease of tubular apoptosis at T0 and T72 either in PMX or CONV group (Fig. 1b).
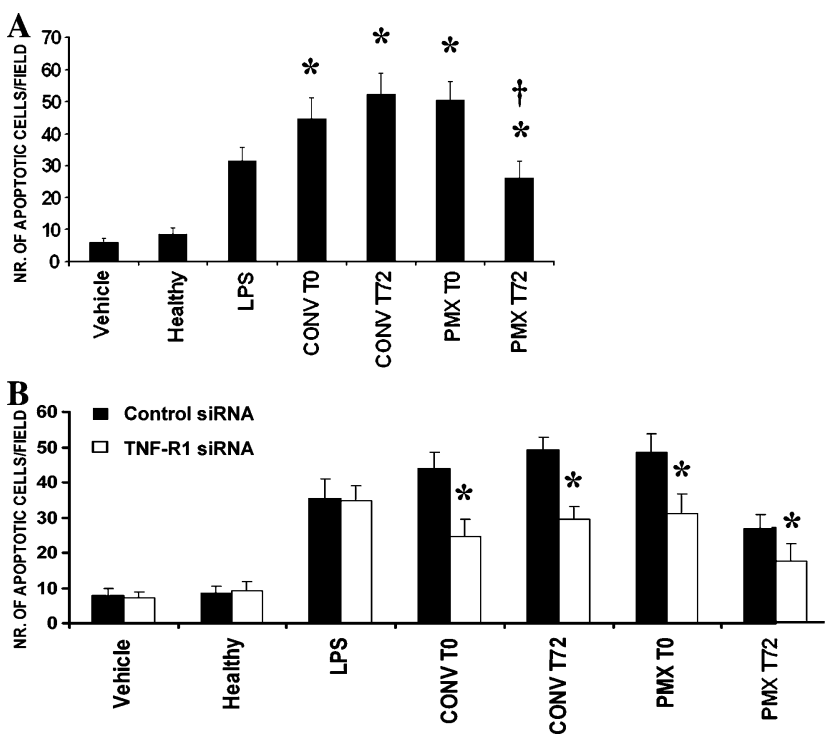

Fig. 1 a Evaluation of tubular apoptosis (TUNEL) induced by incubation for $48 \mathrm{~h}$ with CONV or PMX plasmas in tubular cells. All PMX and CONV plasmas induced a significant increase of tubular apoptosis $(* P<0.05)$. Incubation of tubular cells with PMX T72 plasma resulted in a significant decrease of apoptosis compared to PMX T0 plasma $\left({ }^{\dagger} P<0.05\right)$. LPS $(30 \mathrm{ng} / \mathrm{mL})$ was used as positive control. b Evaluation of tubular apoptosis (TUNEL) in tubular cells subjected to short interfering RNA (siRNA) for tumor necrosis factor-receptor 1 (TNF-R1) or for a noncoding control after incubation with CONV and PMX plasma. Compared to control siRNA, a significant decrease of tubular apoptosis was observed in siRNA TNF-R1 tubular cells incubated with CONV and PMX plasma $(* P<0.05)$. LPS $(30 \mathrm{ng} / \mathrm{mL})$ was used as positive control

Activities of caspase- 3 , caspase- 8 , and caspase- 9 were significantly increased in tubular cells after incubation with PMX T0 and CONV T0 plasma (Fig. 2). All caspases remained activated after incubation of tubular cells with CONV T72 plasma. By contrast, we observed a significant reduction of all caspase activities after PMX T72 plasma challenge.

Compared to controls, FACS and immunofluorescence demonstrated a marked upregulation of Fas expression in presence of PMX T0 and CONV T0 plasma, which remained upregulated after incubation of tubular cells with CONV T72 plasma. A significant decrease in Fas expression was observed after incubation with PMX T72 plasma (Fig. 2). Moreover, tubular cells exposed to PMX T72 plasma showed a significant decrease of the ratio between the mitochondrial proteins $\mathrm{Bax}$ and $\mathrm{Bcl}-2$ (Fig. 2).

Effects on cellular function

After incubation with PMX T0, CONV T0, or CONV T72 plasmas, tubular cells exhibited significantly lower TER values in comparison to stimulation with vehicle alone or 
Fig. 2 Top: Enzyme-linked immunoabsorbent (ELISA) evaluation of caspase-3, caspase- 8 , and caspase- 9 activities on tubular cells cultured for $48 \mathrm{~h}$ with $5 \%$ T0 and CONV T0 and T72 plasmas induced a significant ( $* P<0.05$ vs. healthy plasma). A significant decrease of all caspase activities was found with PMX T72 plasma compared to PMX T0 ${ }^{\dagger} P<0.05$ PMX T72 vs. PMX T0); however, caspase-3 and caspase- 9 activities remained significantly higher than healthy plasma $(* P<0.05$ PMX T72 vs. healthy plasma). Middle: Representative images of FACS and immunofluorescence (insets) analysis of Fas (CD95) expression on tubular cell surface after exposure to CONV CONV T0 and T72 plasmas all induced a marked upregulation of Fas, which was significantly reduced in presence of PMX T72 plasma. $\times 400$

Representative western blot analysis of the mitochondrial proteins $\mathrm{Bax}$ and $\mathrm{Bc} 2$ in tubular cells exposed to $\mathrm{CONV}$ or PMX plasma, and related densitometric analysis expressed as $\mathrm{Bax} / \mathrm{Bcl} 2$ ratio. PMX T0, CONV T0, and CONV T72 plasmas induced a marked upregulation of the $\mathrm{Bax} / \mathrm{Bcl} 2$ ratio that was reduced in the presence of PMX T72 plasma. (Lane 1 Vehicle; Lane 2 Healthy; Lane 3 PMX T0; Lane 4 PMX T72; Lane 5 CONV T0; Lane 6 CONV T72). Beta-actin was used as reference for protein loading CONV or PMX plasma. PMX increase of all caspase activities or PMX plasma. PMX T0 and

magnification. Bottom:
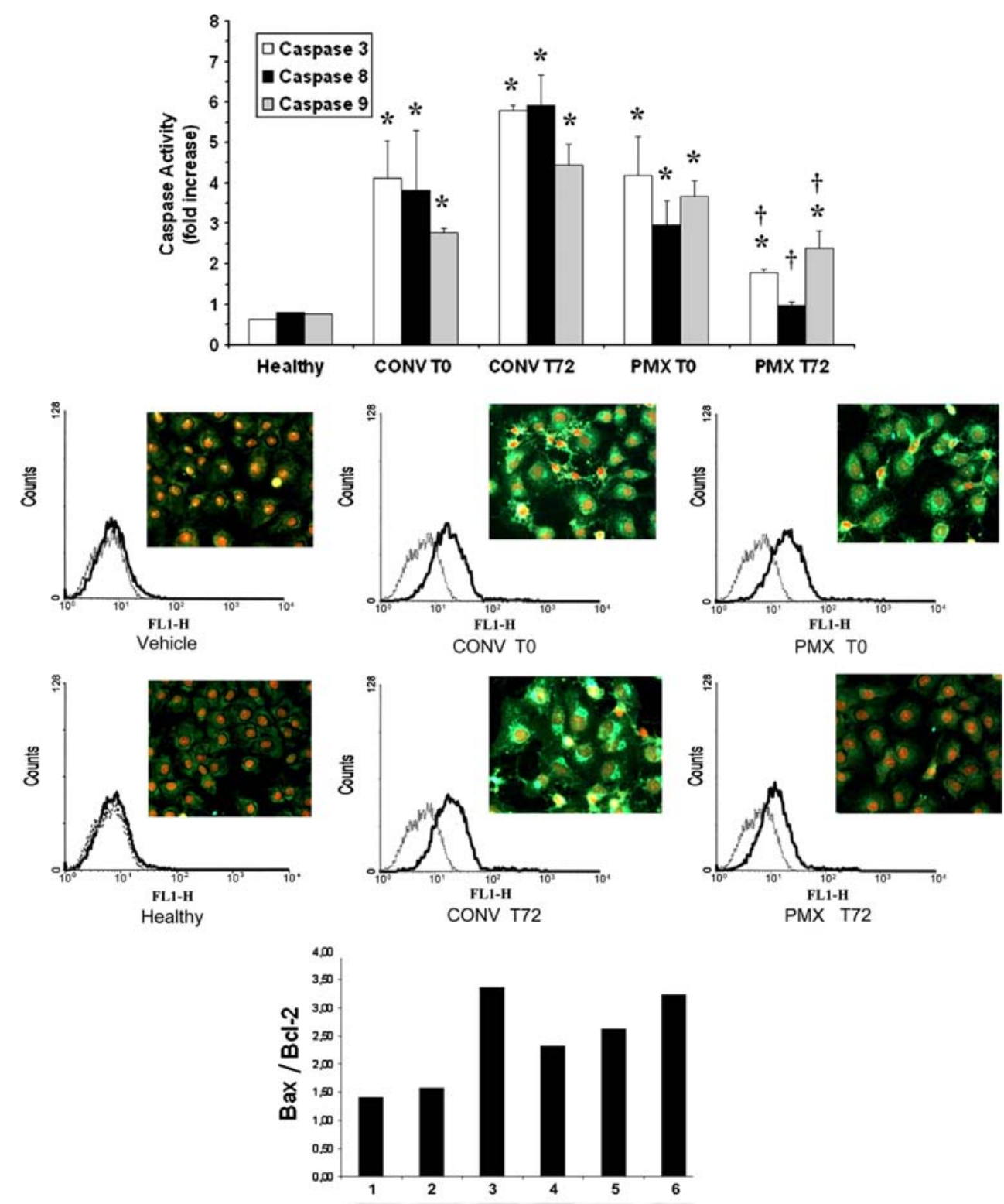

Bax

$\mathrm{Bcl}-2$

$\beta$-actin

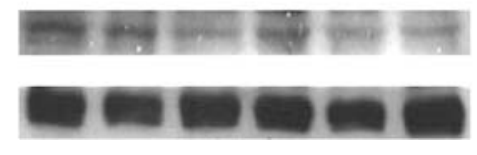

healthy plasma. By contrast, incubation with PMX T72 plasma induced a significant increase of TER levels in comparison to PMX T0, CONV T0, or CONV T72 (Figure 2, $\mathrm{A}$ in ESM).

Incubation of tubular cells with PMX T0, CONV T0, or CONV T72 plasmas resulted in a significant reduction of cell adhesion to type IV collagen-coated, fibronectincoated, and Matrigel-coated surfaces, which was significantly abated in presence of PMX T72 plasma (Figure 2, $\mathrm{B}$ in ESM). Furthermore, we found that CONV plasmas

inhibited morphogenesis of tubular cells cultured onto Matrigel and that PMX-B hemoperfusion restored proper tubular formation (Figure 2, C in ESM).

Incubation with PMX T0 and CONV T0 plasmas induced a significant upregulation of the costimulatory molecule CD40 and of the adhesion receptor ICAM-1 on tubular cell surface, in comparison to exposure to vehicle alone or healthy plasma. The overexpression of both proteins was reduced after incubation with PMX T72 (Figure 3 in ESM). 
Compared to incubation with vehicle or healthy plasma, we observed an intense downregulation of megalin and ZO1 after challenging with PMX T0, CONV T0, or CONV T72 plasmas (Fig. 3 and Figure 4 in ESM). By contrast, PMX T72 plasma did not induce alterations of megalin and ZO1 expression.

Incubation with PMX T0, CONV T0, or CONV T72 plasmas induced a significant reduction of TER (Figure 5, $\mathrm{A}$ in ESM) and a concomitant increase of permeability to albumin (Figure 5, B in ESM) in cultured podocytes. Additionally, PMX T72 plasma inhibited the reduction of TER and albumin diffusion induced by septic plasma. The expression of nephrin was markedly downregulated in the presence of PMX T0, CONV T0, or CONV T72 plasmas (Fig. 4 and Figure 5C in ESM). In contrast, PMX T72 plasma did not alter nephrin expression.

Podocytes expressed basal levels of B7.1 in the presence of vehicle alone or healthy plasma. B7.1 was markedly upregulated only after incubation with PMX T0, CONV T0, or CONV T72 plasmas but not with PMX T72 plasma (Fig. 4 and Figure 6 in ESM).

Furthermore, in correlation to the described results on kidney cell functions, at T72, the PMX group showed a significant reduction of proteinuria and of tubular enzymes not detectable in the CONV group (Figure 7 in ESM).

\section{Discussion}

Direct hemoperfusion through a column of immobilized PMX-B has been proposed to prevent kidney dysfunction in patients with Gram-negative sepsis $[8,33]$. The present study shows that the improvement of renal function observed after PMX-B hemoperfusion might be associated with the attenuation of the proapoptotic activity exerted by circulating factors present in plasma of patients with Gram-negative sepsis. The present study excluded patients with septic shock and/or sepsis with two

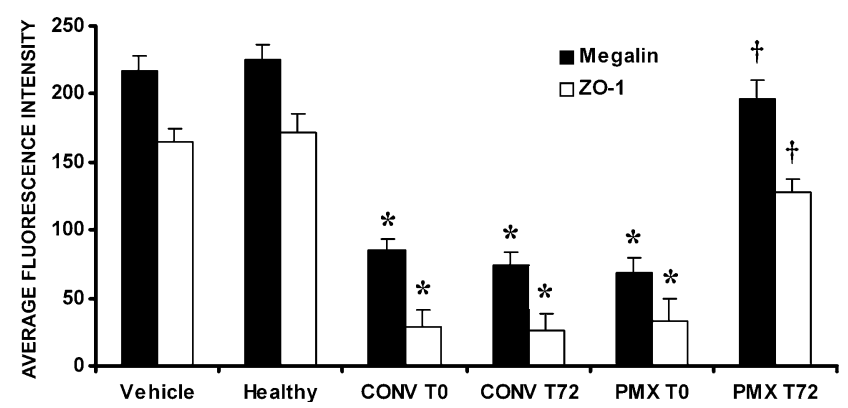

Fig. 3 Average fluorescence expression of megalin and ZO1 on tubular cells. PMX T0, CONV T0, and CONV T72 plasmas induced a marked reduction of megalin and ZO1 expression ( $P<0.05$ vs. healthy plasma). After incubation with PMX T72 plasma, a significant increase of megalin and ZO1 staining was observed $\left({ }^{\dagger} P<0.05\right.$ PMX T72 vs. PMX T0)

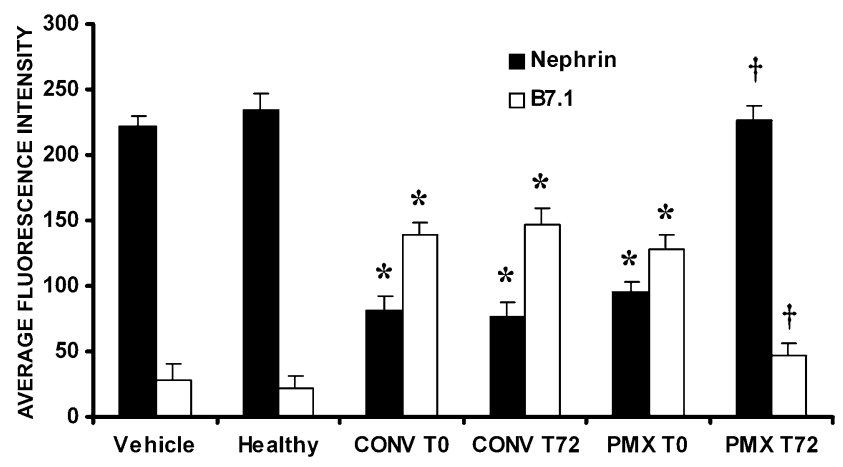

Fig. 4 Average fluorescence expression of nephrin and B7.1 on glomerular podocytes. PMX T0, CONV T0, and CONV T72 plasmas induced a marked reduction of nephrin and the upregulation of B7.1 $(* P<0.05$ vs. healthy plasma). After incubation with PMX T72 plasma, an increased expression of nephrin and a reduced staining for B7.1 was observed ( ${ }^{\dagger} P<0.05$ PMX T72 vs. PMX T0)

or more failing organs, since activated protein $\mathrm{C}$ might be indicated for these patients and data on the interaction between activated protein $\mathrm{C}$ and extracorporeal PMX-B treatment are not currently available [24].

$\mathrm{ARF}$, one of the most frequent organ dysfunction in sepsis [1], has been closely linked to apoptosis [34]. A recent study shows that in Gram-negative sepsis, LPS can directly cause apoptosis of tubular cells through a Fasmediated and caspase-mediated pathways; increased plasma levels of soluble Fas has been described in septic patients [4]. Additionally, experimental models of sepsis have shown that increased caspase activation is associated with the presence of ARF [5]. We found that the reduced proapoptotic activity of plasma on renal tubular cells caused by PMX-B hemoperfusion was mediated by the reduction of Fas upregulation, caspase activity, and Bax/ Bcl2 protein ratio. These results suggest that PMX-B treatment of septic patients decreases the apoptosis of renal epithelial cells through the alteration of the deathreceptor and mitochondrial-associated apoptotic signaling pathways. However, PMX-B hemoperfusion did not completely abrogate the proapoptotic effect of septic plasma on tubular cells, suggesting that circulating mediators other than LPS may be involved in kidney injury. Cunningham and coworkers [36] demonstrated that TNF-alpha is a key mediator of LPS-induced kidney injury acting through TNF-R1 receptor. In this study, we confirmed that PMX-B hemoperfusion induced a significant decrease of plasmatic levels of LPS and TNF- $\alpha$. Even though PMX-B can alter plasmatic lipoprotein profile therefore modulating LPS clearance [37], the results of this study showed a strong correlation between the reduced levels of LPS and the plasma-induced tubular apoptosis. Moreover, we found that TNF-R1 knockdown of tubular cells by siRNA significantly reduced plasmainduced apoptosis also after PMX-B hemoperfusion, confirming a potential prominent role of TNF- $\alpha$ in sepsisrelated kidney injury. 
Recent clinical studies showed that in patients with Gram-negative sepsis, compared to conventional treatment, hemoperfusion through a PMX-B cartridge significantly decreases vasopressors requirement [9] and reduces the need for continuous renal replacement therapy (CRRT) [9, 12]. Consistent with previous studies, we observed that $72 \mathrm{~h}$ after entering the study, patients included in the PMX group had a significant reduction of catecholamine requirement, an improvement of SOFA and RIFLE scores, and a significant decrease in RRT requirement as compared to the CONV group. In septic patients with endotoxemia, Nakamura and coworkers recently showed a relevant improvement in renal function after treatment with PMX-B hemoperfusion; this functional improvement was associated to a significant decrease of urine NAG/creatinine ratio, albuminuria, and plasma endotoxin level $[12,35]$. Consistently, we found that $72 \mathrm{~h}$ after entering the study, the PMX group showed a significant reduction of the urinary protein-to-creatinine ratio and of the tubular enzymes GGT, NAG, AAP.

The alteration of cellular permeability and polarity can contribute to kidney dysfunction [36, 37]. In this study, we show that in tubular cells, the plasma taken from the patients of the CONV group at T0 and T72 and of the PMX group at T0 induced a marked decrease of the tight junction molecule ZO1. By contrast, plasma of patients treated with PMX-B after $72 \mathrm{~h}$ did not exhibit this effect. Moreover, while the plasma taken from patients of the CONV group turned down the expression of megalin (the endocytic receptor that regulates the physiological reabsorption of glomerular-filtered low molecular weight proteins), the plasma drawn from patients treated with PMX-B left unchanged in its expression. These results suggest that PMX-B treatment may remove factors able to alter physiologic kidney cell polarity and protein handling.

Microalbuminuria is an early index of impairment of glomerular permeability in septic patients [38]. We observed that albumin diffusion across podocyte monolayer decreased only in the presence of plasma collected after PMX-B treatment. This phenomenon was associated with the maintenance of a normal expression of nephrin, a slit diaphragm protein known to modulate glomerular permeability, that significantly downregulated after incubation with CONV T0, CONV T72, and PMX T0 plasma. Moreover, CONV T0, CONV T72, and PMX T0 plasma induced the upregulation of B7.1, a costimulatory molecule, which is known to be induced by LPS through TLR-4 signaling [32]. This effect was almost completely blunted in presence of plasma collected after PMX-B hemoperfusion, thus suggesting a further protective action of this depurative treatment on the development of proteinuria and in the extension of renal injury.

In conclusion, this study demonstrates that extracorporeal therapy with PMX-B reduces the proapoptotic activity of the plasma of septic patients on cultured cell. These data confirm the role of apoptosis in the development of sepsis-related acute renal dysfunction and indicate a putative protective effect of PMX-B hemoperfusion in the early prevention of kidney injury.

Open Access This article is distributed under the terms of the Creative Commons Attribution Noncommercial License which permits any noncommercial use, distribution, and reproduction in any medium, provided the original author(s) and source are credited.

\section{References}

1. Schrier RW, Wang W (2004) Acute renal failure and sepsis. N Engl J Med 351:159-169

2. Angus DC, Linde-Zwirble WT, Lidicker J, Clermont G, Carcillo J, Pinsky MR (2001) Epidemiology of severe sepsis in the United States: analysis of incidence, outcome, and associated costs of care. Crit Care Med 29:1303-1310

3. Cunningham PN, Wang Y, Guo R, He G, Quigg RJ (2004) Role of Toll-like receptor 4 in endotoxin-induced acute renal failure. J Immunol 172:2629_ 2635

4. Jo SK, Cha DR, Cho WY, Kim HK, Chang KH, Yun SY, Won NH (2002) Inflammatory cytokines and lipopolysaccharide induce Fas-mediated apoptosis in renal tubular cells. Nephron 91:406-415
5. Guo R, Wang Y, Minto AW, Quigg RJ, Cunningham PN (2004) Acute renal failure in endotoxemia is dependent on caspase activation. J Am Soc Nephrol 15:3093-3102

6. Hotchkiss RS, Swanson PE, Freeman BD, Tinsley KW, Cobb JP, Matuschak GM, Buchman TG, Karl IE (1999) Apoptotic cell death in patients with sepsis, shock, and multiple organ dysfunction. Crit Care Med 27:12301251

7. Linton AL, Walker JF, Lindsay RM, Sibbald WJ (1985) Acute renal failure and tubular damage due to sepsis in an animal model. Proc Eur Dial Transplant Assoc Eur Ren Assoc 21:837-842

8. Shoji H (2003) Extracorporeal endotoxin removal for the treatment of sepsis: endotoxin adsorption cartridge (Toraymyxin). Ther Apher Dial 7:108114
9. Vincent JL, Laterre PF, Cohen J, Burchardi H, Bruining H, Lerma FA, Wittebole X, De Backer D, Brett S, Marzo D, Nakamura H, John S (2005) A pilot-controlled study of a polymyxin B-immobilized hemoperfusion cartridge in patients with severe sepsis secondary to intra-abdominal infection. Shock 23:400-405

10. Nakamura T, Ebihara I, Shimada N, Koide H (1998) Changes in plasma erythropoietin and interleukin-6 concentrations in patients with septic shock after hemoperfusion with polymyxin B-immobilized fiber. Intensive Care Med 24:1272-1276

11. Cruz DN, Ronco C (2007) Acute kidney injury in the intensive care unit: current trends in incidence and outcome. Crit Care 11:149 
12. Nakamura T, Kawagoe Y, Matsuda T, Ueda Y, Koide H (2004) Effects of polymyxin $\mathrm{B}$ immobilized fiber on urinary $\mathrm{N}$-acetyl-beta-glucosaminidase in patients with severe sepsis. ASAIO J 50:563-567

13. Wesche-Soldato DE, Swan RZ, Chung CS, Ayala A (2007) The apoptotic pathway as a therapeutic target in sepsis. Curr Drug Targets 8:493-500

14. Kang YH, Falk MC, Bentley TB, Lee CH (1995) Distribution and role of lipopolysaccharide in the pathogenesis of acute renal proximal tubule injury. Shock 4:441-449

15. Bordoni V, Bolgan I, Brendolan A, Crepaldi C, Gastaldon F, D'Intini V, Pilotto L, Inguaggiato P, Bonello $\mathrm{M}$, Galloni E, Everard P, Bellomo R, Ronco C (2003) Caspase-3 and -8 activation and cytokine removal with a novel cellulose triacetate superpermeable membrane in an in vitro sepsis model. Int $\mathbf{J}$ Artif Organs 26:897-905

16. Ayala A, Herdon CD, Lehman DL, DeMaso CM, Ayala CA, Chaudry IH (1995) The induction of accelerated thymic programmed cell death during polymicrobial sepsis: control by corticosteroids but not tumor necrosis factor. Shock 3:259-267

17. Crouser ED, Julian MW, Weinstein DM, Fahy RJ, Bauer JA (2000) Endotoxin-induced ileal mucosal injury and nitric oxide dysregulation are temporally dissociated. Am J Respir Crit Care Med 161:1705-1712

18. Camussi G, Mariano F, Biancone L, De Martino A, Bussolati B, Montrucchio G, Tobias PS (1995) Lipopolysaccharide binding protein and CD14 modulate the synthesis of platelet-activating factor by human monocytes and mesangial and endothelial cells stimulated with lipopolysaccharide. J Immunol 155:316-324

19. Imai Y, Parodo J, Kajikawa O, de Perrot M, Fischer S, Edwards V, Cutz E, Liu M, Keshavjee S, Martin TR, Marshall JC, Ranieri VM, Slutsky AS (2003) Injurious mechanical ventilation and end-organ epithelial cell apoptosis and organ dysfunction in an experimental model of acute respiratory distress syndrome. JAMA 289:21042112

20. Hotchkiss RS, Karl IE (2003) The pathophysiology and treatment of sepsis. N Engl J Med 348:138-150
21. Pasero D CV, Assenzio B, Puntorieri V, Mascia L, Ranieri VM (2006) Hemoperfusion with Polymyxin-B Cartridge protecs from sepsis-related acute kidney injury. Intensive Care Med 32:S224

22. Levy MM, Fink MP, Marshall JC, Abraham E, Angus D, Cook D, Cohen J, Opal SM, Vincent JL, Ramsay G (2003) $2001 \mathrm{SCCM} / \mathrm{ESICM} / \mathrm{ACCP} /$ ATS/SIS International Sepsis Definitions Conference. Intensive Care Med 29:530-538

23. Bernard GR, Vincent JL, Laterre PF, LaRosa SP, Dhainaut JF, LopezRodriguez A, Steingrub JS, Garber GE, Helterbrand JD, Ely EW, Fisher CJ Jr (2001) Efficacy and safety of recombinant human activated protein $\mathrm{C}$ for severe sepsis. N Engl J Med 344:699-709

24. Dellinger RP, Carlet JM, Masur H, Gerlach H, Calandra T, Cohen J, GeaBanacloche J, Keh D, Marshall JC, Parker MM, Ramsay G, Zimmerman JL, Vincent JL, Levy MM (2004) Surviving Sepsis Campaign guidelines for management of severe sepsis and septic shock. Crit Care Med 32:858873

25. Moreno R, Vincent JL, Matos R, Mendonca A, Cantraine F, Thijs L, Takala J, Sprung C, Antonelli M, Bruining H, Willatts S (1999) The use of maximum SOFA score to quantify organ dysfunction/failure in intensive care. Results of a prospective, multicentre study. Working Group on Sepsis related Problems of the ESICM. Intensive Care Med 25:686-696

26. Bellomo R, Kellum JA, Ronco C (2007) Defining and classifying acute renal failure: from advocacy to consensus and validation of the RIFLE criteria. Intensive Care Med 33:409-413

27. Xin G, Wang M, Jiao LL, Xu GB, Wang HY (2004) Protein-to-creatinine ratio in spot urine samples as a predictor of quantitation of proteinuria. Clin Chim Acta 350:35-39

28. Conaldi PG, Bottelli A, Baj A, Serra C, Fiore L, Federico G, Bussolati B, Camussi G (2002) Human immunodeficiency virus- 1 tat induces hyperproliferation and dysregulation of renal glomerular epithelial cells. Am J Pathol 161:53-61

29. Conaldi PG, Biancone L, Bottelli A, Wade-Evans A, Racusen LC, Boccellino M, Orlandi V, Serra C, Camussi G, Toniolo A (1998) HIV-1 kills renal tubular epithelial cells in vitro by triggering an apoptotic pathway involving caspase activation and Fas upregulation. J Clin Invest 102:20412049
30. Biancone L, Cantaluppi V, Segoloni G, Boccellino M, Del Sorbo L, Conaldi PG, Tjoelker LW, Maruyama S, Cantu E, Stern D, Andres G, Camussi G (2000) Role of platelet-activating factor in functional alterations induced by xenoreactive antibodies in porcine endothelial cells. Transplantation 70:1198-1205

31. Glynne PA, Picot J, Evans TJ (2001) Coexpressed nitric oxide synthase and apical beta(1) integrins influence tubule cell adhesion after cytokine-induced injury. J Am Soc Nephrol 12:23702383

32. Reiser J, von Gersdorff G, Loos M, Oh J, Asanuma K, Giardino L, Rastaldi MP, Calvaresi N, Watanabe H, Schwarz K, Faul C, Kretzler M, Davidson A, Sugimoto H, Kalluri R, Sharpe AH, Kreidberg JA, Mundel P (2004) Induction of B7-1 in podocytes is associated with nephrotic syndrome. J Clin Invest 113:1390-1397

33. Nemoto H, Nakamoto H, Okada H, Sugahara S, Moriwaki K, Arai M, Kanno Y, Suzuki H (2001) Newly developed immobilized polymyxin B fibers improve the survival of patients with sepsis. Blood Purif 19:361-368; discussion 368-369

34. Wan L, Bellomo R, Di Giantomasso D, Ronco C (2003) The pathogenesis of septic acute renal failure. Curr Opin Crit Care 9:496-502

35. Trof RJ, Di Maggio F, Leemreis J, Groeneveld AB (2006) Biomarkers of acute renal injury and renal failure. Shock 26:245-253

36. Tryggvason K, Patrakka J, Wartiovaara J (2006) Hereditary proteinuria syndromes and mechanisms of proteinuria. N Engl J Med 354:13871401

37. Christensen EI, Birn H (2001) Megalin and cubilin: synergistic endocytic receptors in renal proximal tubule. Am J Physiol Renal Physiol 280:F562-573

38. De Gaudio AR, Adembri C, Grechi S, Novelli GP (2000) Microalbuminuria as an early index of impairment of glomerular permeability in postoperative septic patients. Intensive Care Med 26:1364-1368 